\title{
ADAPTATION TO A MOTION-BASED AND NON-MOTION-BASED SIMULATOR
}

\author{
Renee F. Slick, Tuan Q. Tran, Elizabeth T. Cady \\ Department of Psychology \\ Kansas State University \\ Manhattan, Kansas, USA \\ E-mail: $\underline{\text { rslick@ } a \text { ksu.edu }}$
}

\begin{abstract}
Summary: This study ( $\mathrm{N}=129$, including 59 males and 61 females) examined the issue of realism and motion sickness between motion-based and non-motionbased simulators. Specifically, this research address whether enhancing a driving simulator with motion capabilities increases the realism of the simulator and, if so, does this increase in subjective realism increase participants' vulnerability to motion sickness. Approximately half of the participants drove a motion-based simulator while the other half drove a non-motion-based simulator on four independent drives within an experimental session. Results showed that the motion-based simulator was rated more realistic than the non-motion-based simulator. However, it was also found that participants in the motion-based simulator had higher negative physical health ratings than participants in the nonmotion-based simulator. Our results suggest that training programs need to consider the trade-off between realism and motion sickness.
\end{abstract}

\section{INTRODUCTION}

High ecological validity is important, not only for actually identifying valid perceptual cues that affect driving performances but also for generalizing laboratory results (such as training performances) to the real world. Hence, the issue of realism and generalizability is unquestionably important to simulator studies. According to Manser and Hancock (1996), realism can be defined as how well the environment in the simulator imitates the outside world. In other words, does the simulator contain an accurate reflection of the realistic environmental cues? In addition, generalizability can be defined as "the ability to extend the results obtained through tests to other populations and environments" (Manser and Hancock, 1996). In other words, how effective are laboratory results and can they be extended to the real-world? Arguably, for laboratory studies to successfully generalize their results (e.g., training) to the realworld, they first must accurately capture the "realism" of the construct of interest. This is most true in driving simulation. However, one unresolved question is whether enhancing the realism of simulation research may have inadvertent negative physical or health consequences to the trainee.

The purpose of this study was to investigate whether enhancement of a driving simulator by equipping the simulator with motion will have negative physical or health consequences compared to a non-motion-based driving simulator. 


\section{METHOD}

\section{Participants}

One hundred and twenty participants (59 males and 61 females) were recruited for the present study from Kansas State University and a local high school.

\section{Materials}

Participants were given questionnaires to complete prior to the beginning of the first drive and after each individual drive. Prior to the first drive, participants were given a Motion History Questionnaire and a Pre-Drive General Health Questionnaire. The Motion History Questionnaire was used to assess a person's susceptibility to motion sickness in terms of past experience with motion sickness in both simulated and real environments. In addition, a PreDrive General Health Questionnaire, was used to assess how the person feels at that moment on a number of dimensions, with the critical question "Are you feeling sick today?" The questionnaire also listed 16 physical symptoms: "general discomfort," "fatigue," "headache," "eyestrain," "difficulty focusing," "increased salivation," "sweating," "nausea," "difficulty concentrating," "pressure in the head," "blurred vision," “dizzy (eyes open), " dizzy (eyes closed)," "loss of upright orientation," "stomach awareness," and "burping." Participants indicated the degree to which each adjective best described their current experiences on a 4-point response scale with the following anchors: "None," "Slight," "Moderate," and "Severe."

After each simulated drive, participants completed a General Health Questionnaire and a Simulation Face Validity Measure. To ensure that participants were not developing adverse physical symptoms from the simulated drive, participants completed a General Health Questionnaire (SSQ). The General Health Questionnaire (SSQ) is identical to the Pre-Drive General Health Questionnaire except the question "Are you feeling sick today?" is omitted. Finally, a Simulator Face Validity Measure (SFVM) was used to assess the realism of various aspects of the stimulated drives on a 5-point bi-polar scale. This questionnaire included questions regarding the scenery (e.g., How did you feel about the scenery (trees, buildings, etc.)?) and traffic level in the specific drive (e.g., How was the traffic level?) as well as questions regarding the experience of driving (e.g., How realistic is the steering?).

\section{Procedures}

Participants were independently tested individually or in pairs of two. When tested in pairs, participants alternated between the simulated drives and other activites as part of a larger study. Upon entering the lab and after completing their consent forms, participants were given both the motion history and the pre-drive genaral health questionnaries to complete. Any individuals who responded "yes" to the critical question "Are you feeling sick today?" on the pre-drive general health questionnaire were excused and dimissed from continuing with the experiment. Participants completed four drives that increased in complexity over the time period. To adapt the participants to the simulator, Drive 1 simply directed participant to drive in straight path. Drive 2 increase the complexity by including wide left and right turns. Drive 3 instructed participants to drive in a simulated neighborhood with sharper left and right turns, as well as with low-level traffic. Finally, Drive 4 consisted of sharp turns and driving through a moderate level 
of traffic. After each of the drives, participants were given both the general health questionnaire and simulator face validity measure to complete to measure their current feelings and opinions toward the simulator. In addition, any participants who rated any of the 16 adjectives on the general health questionnaire as "severe" or any participants who reported intense feeling of nausea were dismissed from the experiment. After completing the four drives and questionnaires, participants were thanked, debriefed, and dismissed. The experiment lasted approximately two hours for participants tested in pairs and one hour for participants tested alone.

\section{RESULTS}

\section{General Health Questionnaire}

For each of the 16 adjectives on the pre-drive general health questionnaire and on the general health questionnaire, the ordinal scale was dummy coded with "none" given a one, "slight" a two, "moderate" a three, and "severe" a four. Ideally, participants should remain at their baseline level of discomfort throughout the session. If a participant had increased scores across drives in a session, this indicated that the participant was experiencing some amount of simulator sickness.

To assess the participants' level of physical discomfort between a motion-based simulator and a non-motion-based simulator during each simulator drive, as well as examine whether gender modulates such an effect, participants' 16 adjective ratings were averaged across each drive and submitted to a 5 (time: pre, drive1, drive2, drive3, drive4) X 2(motion: motion, no motion) X 2 (gender: male, female) repeated measure analysis of variance (ANOVA) with motion and gender being the between-subject variable. A main effect of time and motion was found to be statistically significant, $F(1,116)=17.70, p<.05$ and $F(1,116)=14.85, p<.05$, respectively but gender was not significant $(\mathrm{p}=.08)$. However, the main effect of time and motion was mediated by a statistically significant interaction between time and motion, $F(1,116)=17.54, p<.05$. As Figure 1 displays, a consistent trend emerged, as participants completed each of their four drives, those participants in the motion-based simulators reported that they are getting progressively worse, whereas those participants in the non-motion-based simulator tended to observe few or no changes in their ratings. Finally, although a main effect of gender was not observed, as shown in Figure 2, females consistently showed higher SSQ ratings across each of the four drives.

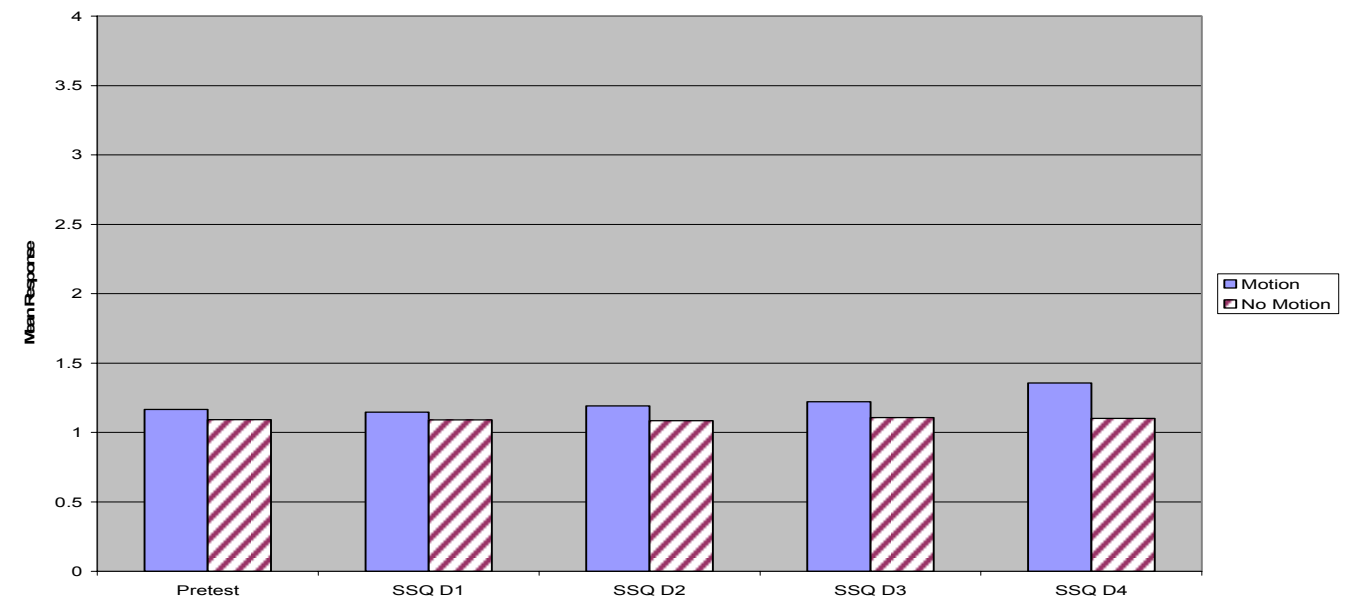

Figure 1. Motion and Non-Motion-Based SSQ Ratings as a Function of Number of Drives 


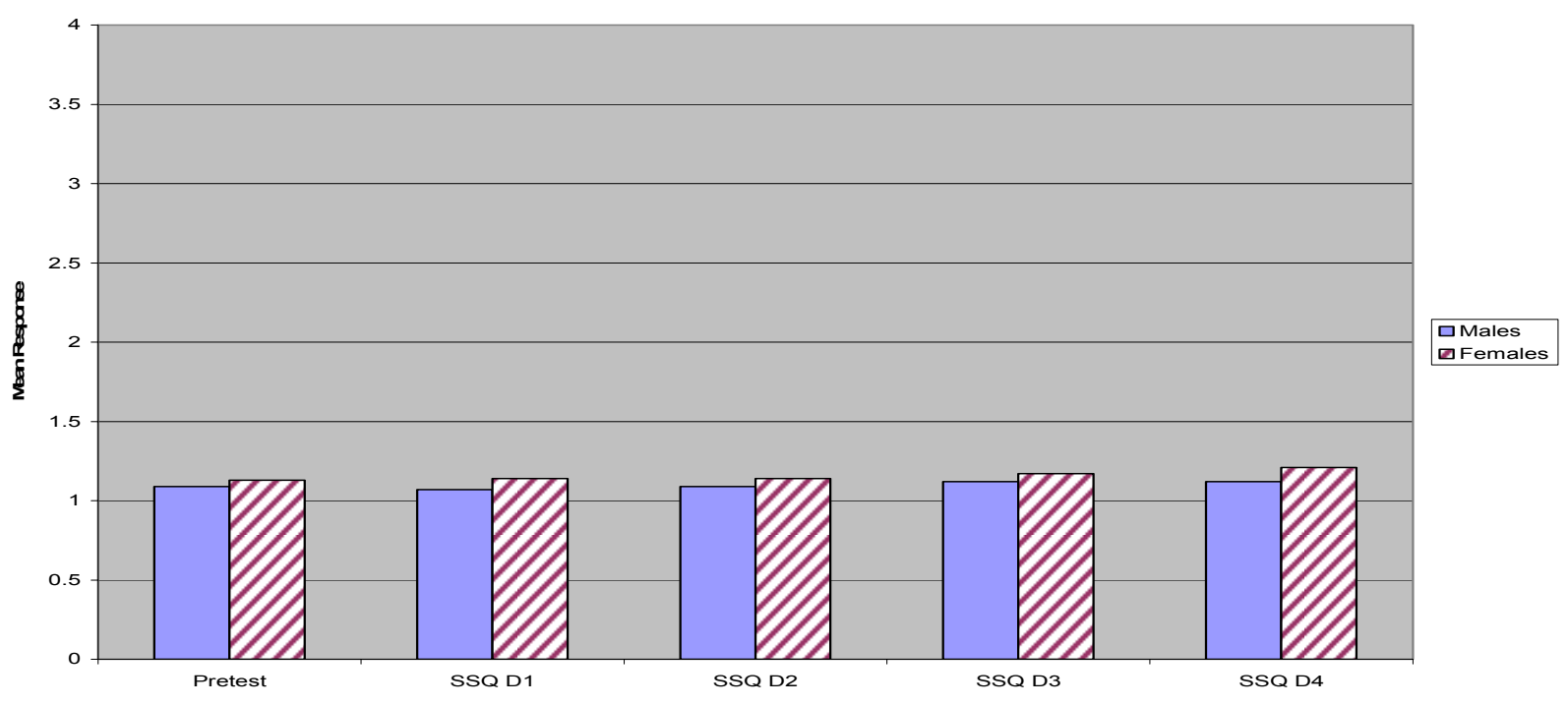

Figure 2. Males and Females SSQ Ratings as a Function of Number of Drives

To further investigate and target the specific adjective of the physical discomfort between motion-based simulator and non-motion-based simulator, a separate 5 (time: pre, drive1, drive2, drive3, drive4) X 2(motion: motion, no motion) X 2(gender: male, female) repeated measure analysis of variance (ANOVA) was performed on each of the 16 adjectives. The analysis showed a statistically significant of time $\mathrm{x}$ motion interaction for 14 of the 16 physical discomfort adjectives $(\mathrm{p}<.05)$ as shown in Table 1 . This suggests that as the number of drives increased, participants' level of physical discomfort increased in general not just specifically one particular symptom.

Table 1. Significant Level of the 16 Physical Discomfort Adjectives Listed in SSQ

\begin{tabular}{|l|c|l|c|}
\hline Symptoms & Significant Level & Symptoms & $\begin{array}{l}\text { Significant } \\
\text { Level }\end{array}$ \\
\hline General discomfort & $\mathrm{p}<.05$ & Difficulty concentrating & $\mathrm{p}<.05$ \\
\hline Fatigue & $\mathrm{p}<.05$ & Pressure in the head & $\mathrm{p}<.05$ \\
\hline Headache & $\mathrm{p}=\mathrm{n} . \mathrm{s}$. & Blurred vision & $\mathrm{p}<.05$ \\
\hline Eyestrain & $\mathrm{p}<.05$ & Dizzy (eyes open) & $\mathrm{p}<.05$ \\
\hline Difficulty focusing & $\mathrm{p}<.05$ & Dizzy (eyes closed) & $\mathrm{p}<.05$ \\
\hline Increased salivation & $\mathrm{p}<.05$ & Loss of upright orientation & $\mathrm{p}<.05$ \\
\hline Sweating & $\mathrm{p}<.05$ & Stomach awareness & $\mathrm{p}<.05$ \\
\hline Nausea & $\mathrm{p}<.05$ & Burping & $\mathrm{p}=$ n.s. \\
\hline
\end{tabular}

\section{Simulator Face Validity Measure (SFVM)}

Questions 1, 8, and 10 in the drive face-validity questionnaire given after each simulator drive, as well as question 5 in the final simulator face-validity questionnaire that was given at the end of the experimental day, consisted of dichotomous responses; hence, these data were submitted to Pearson's chi-square significant testing. The remaining responses to questions in both the drive 
face-validity and final simulator face-validity questionnaires were analyzed using analysis of variance (ANOVA).

A 5 (time: pre, drive1, drive2, drive3, drive4) X 2(motion: motion, no motion) X 2(gender: male, female) repeated measure analysis of variance (ANOVA) on the face-validity found participants in the motion-based simulator reported higher mean ratings compared to participants in the nonmotion-based simulator for question 3 ("How was the traffic level?"), $F(1,116)=7.71, p<.05$. Such significant results suggest that motion increases the realism of the simulator driving experience. Finally, a Pearson's chi-square revealed not statistically signficant results for dichotomous responses in Question 1, 8, and 10.

\section{CONCLUSIONS}

Overall, our study found that having a motion-based simulator enhances some perception of the validity of the simulator (i.e., traffic level) but it comes with a cost in increasing participants' vulnerability to motion sickness. In addition and consistent with a prior study (Park et al., 2004), we did not find a gender effect on degree of vulnerability to motion sickness. These findings are important in terms of simulation design and training where the key focus is to construct a safe simulated environment to develop and train behaviors that may be generalizable to the external real world.

\section{REFERENCES}

Manser, M. P., \& Hancock, P. A. (1996). Influence of approach angle on estimates of time-tocontact. Ecological Psychology, 8(1): 71-99.

Park, G., Rosenthal, T. J., Allen, R. W., Cook, M. L., Fiorentino, D., \& Viirre, E. (2004). Simulator sickness results obtained during a novice driver training study. Proceedings of the Human Factors and Ergonomics Society $48^{\text {th }}$ Annual Meeing, 2652- 2656. 